\title{
Effect of packaging on quality of fresh-cut thyme
}

\author{
C. Zhang, Y Li, Y Wang, Y Ma \& X Zhao
}

Beijing Vegetable Research Center, Beijing Academy of Agriculture and Forestry Sciences; Beijing Key Laboratory of Fruits and Vegetable Storage and Processing; Key Laboratory of Biology and Genetic Improvement of Horticultural Crops (North China), Ministry of Agriculture; Key Laboratory of Urban Agriculture (North), Ministry of Agriculture, 9 Shuguanghuayuan Road, Haidian District, Beijing, China

KEYWORD: fresh cut; thyme; packaging; 1-MCP; sensory

ABSTRACT: The effect of packaging on the quality of fresh-cut thyme was evaluated. The packaging showed no significant influence on the shelf life of the fresh-cut thyme when its shelf life was $7 \mathrm{~d}$. The 1-MCP and modified atmosphere packaging treatments hold the sensory features of the thyme, and avoided the loss of the greenness of the fresh thyme. Moreover, the 1-MCP and modified atmosphere packaging treatment shown no influence of the flavor of the of the fresh-cut thyme.

\section{Introduction}

Thyme (Thymus vulgaris L.) is a subshrub from the lamiaceae family (Fornari, et al., 2012) with plants that are rich in essential oils which contain more than 60 ingredients and antioxidative phenolic substances that have antioxidant properties and antimicrobial activity(Rota, et al., 2008). Thyme was used in human nutrition as a spice and food flavor and as antioxidant for treatment of many diseases (Mathur, et al., 2003).

Packaging is an important consideration for the quality and shelf life of the fresh-cut vegetable (Bennik, et al., 1996, Gil, et al., 2002). Modified atmosphere packaging (MAP) has effectively extended the shelf life of papaya (Waghmare \& Annapure, 2013), pineapple (Rocculi, et al., 2009), watercress (Silveira, et al., 2014) and etc. effectively by lowering physiological metabolism. 1-Methylcyclopropene (1-MCP) has been proven to have enormous benefit to control the ripening and senescence of fruit and vegetables (Watkins, 2006), which has been applied to extend the shelf life of the banana (Vilas-Boas \& Kader, 2006), broccoli (Cefola \& Amodio, 2010), tomato (Kulkarni \& Vijayanand, 2012), avocado (Hershkovitz, et al., 2005) and etc.. Fresh-cut thyme had been widely accepted as a culinary herb for flavouring and seasoning (Omidbeygi, et al., 2007). However, the application of the MAP and 1-MCP on fresh-cut thyme was not found to the best of our knowledge.

In order to extend the shelf life and hold the flavor, the MAP and 1-MCP treatments were applied to the fresh-cut thyme. The shelf life and the flavor of the fresh-cut thyme was compared with the treated thyme.

\section{Material and Methods}

\section{Preparation and treatments of fresh-cut thyme}

Thyme was picked from our Tongzhou farm (Tongzhou District Beijing, 2014). The fresh leaf of the thyme was stored at $4{ }^{\circ} \mathrm{C}$ before use. The thyme was washed by the tap water at $4{ }^{\circ} \mathrm{C}$ to remove the soil and some inclusion. The thyme was further sterilized by $100 \mathrm{ppm}$ sodium hypochlorite to reduce the microflora for $5 \mathrm{~min}$ at $4{ }^{\circ} \mathrm{C}$. The washed thyme was stirred and cut manually.

Control: The cut leaf of about $15 \mathrm{~g}$ was packaged in a $20 \mathrm{~cm} \times 18 \mathrm{~cm}$ polythene film and was stored at $4{ }^{\circ} \mathrm{C}$. 
MAP: The cut leaf of about $15 \mathrm{~g}$ was packaged in a $20 \mathrm{~cm} \times 18 \mathrm{~cm}$ polythene film. The air in the film was replaced by the modified atmosphere (20\% carbon dioxide, and $80 \%$ nitrogen). The fresh-cut thyme was stored at $4{ }^{\circ} \mathrm{C}$.

1-MCP: The cut leaf was packaged in a sealed chamber and was subjected to 1-MCP treatment of $1 \mathrm{ppm}$ for $24 \mathrm{~h}$ at $4{ }^{\circ} \mathrm{C}$. The treated leaf of about $15 \mathrm{~g}$ was packaged in a $20 \mathrm{~cm} \times 18 \mathrm{~cm}$ polythene film and was stored at $4{ }^{\circ} \mathrm{C}$.

\section{Determination of chlorophyll content by spectrophotometry}

To measure the chlorophyll content, $20 \mathrm{ml}$ of $80 \%$ acetone was added to $5 \mathrm{~g}$ of each sample and mixed with a homogenizer (IKA T10 Basic, Werke GmbH \& Co. KG, Staufen, Germany) for 1 min at a high speed, then centrifuged at $8,000 \mathrm{rpm}$ for $5 \mathrm{~min}$ at $4{ }^{\circ} \mathrm{C}$, and then filtered through Whatman No. 1 filter papers. The volume was adjusted to $25 \mathrm{~mL}$ and the absorbance values were measured at 663 and $645 \mathrm{~nm}$ by a spectrophotometer (UV-1800, Shimadzu Corporation, Kyoto, Japan). The chlorophyll content of the sample was calculated followed by Equation 1.

$$
\text { Chlorophyll }=\frac{\left(20.2 \times A_{645}+8.02 \times A_{663}\right) \times D}{1000}
$$

where chlorophyll content is $\mathrm{mg} / \mathrm{g}$ fresh weight; $A_{645}$ and $A_{663}$ are the absorbance at $645 \mathrm{~nm}$ and $663 \mathrm{~nm}$, respectively; $D$ is the dilution factor of the sample.

\section{Total microflora counts}

Samples were serially diluted, plated in total count agar for total microflora counts, followed the recently reported method (Rivasa, et al., 2006). The plates were incubated at $37{ }^{\circ} \mathrm{C}$ for $48 \mathrm{~h}$ and counted manually.

\section{Flavor comparison}

The flavor of the samples was compared by an electronic nose PEN2 (Airsense Analytics GmbH, Schwerin, Germany). The electronic nose was turned on for $30 \mathrm{~min}$ and flushed the testing system for $180 \mathrm{~s}$. The sample of $2 \mathrm{ml}$ was put in the testing tube. And then the electronic sensor was put into the testing tube to collect the results for $60 \mathrm{~s}$. The response of the sensor in 48 52 s were evaluated by a principal component analysis.

\section{Statistical Analysis.}

Analysis of variance (ANOVA) was used to compare mean differences of the results. If the differences in mean existed, multiple comparisons were performed using Duncan's Multiple Range Test. All analysis was conducted using SPSS for Window Version 19. All experiments were done in triplicates or more.

\section{Results and Discussion}

\section{Effect of packaging on shelf life of fresh-cut thyme}

The effect of packaging on the total micorflora count of fresh-cut thyme is shown in Figure 1. Different countries or companies have their own microflora criterion. In Beijing, most company set 5.0 LogCFU/g as the microflora threshold of the fresh-cut vegetable. The total microflora count of the 1-MCP and MAP treatment was similar to that of the control, and exceeded the $5.0 \mathrm{LogCFU} / \mathrm{g}$ in the $7^{\text {th }}$ day. Consequently, the 1-MCP and MAP treatment showed no influence on the shelf life of the thyme. The shelf life of the fresh-cut thyme was $7 \mathrm{~d}$ from the view of the microflora. 


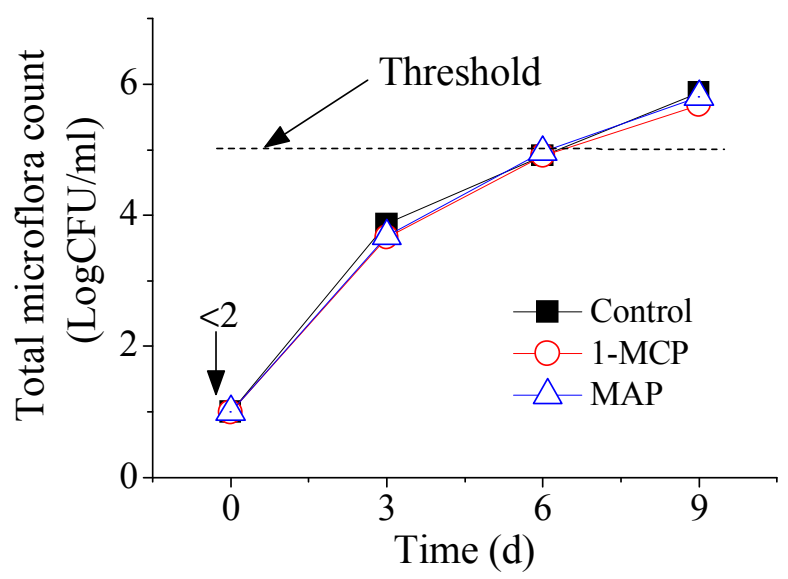

Figure 1 Effect of packaging on the total micorflora count of the fresh-cut thyme

\section{Effect of packaging on the sensory features of fresh-cut thyme}

The sensory features of the fresh thyme were compared with the treated thyme (Figure 2). The stem of the fresh thyme was light red, while that of the control turned yellow. The stem of the MAP and 1-MCP treatment was between the light red and yellow. On the other hand, the leaf of the 1-MCP and MAP was dark green, while the leaf of the control was light green. Therefore, the 1-MCP and MAP treated thyme was more attractive than the control.
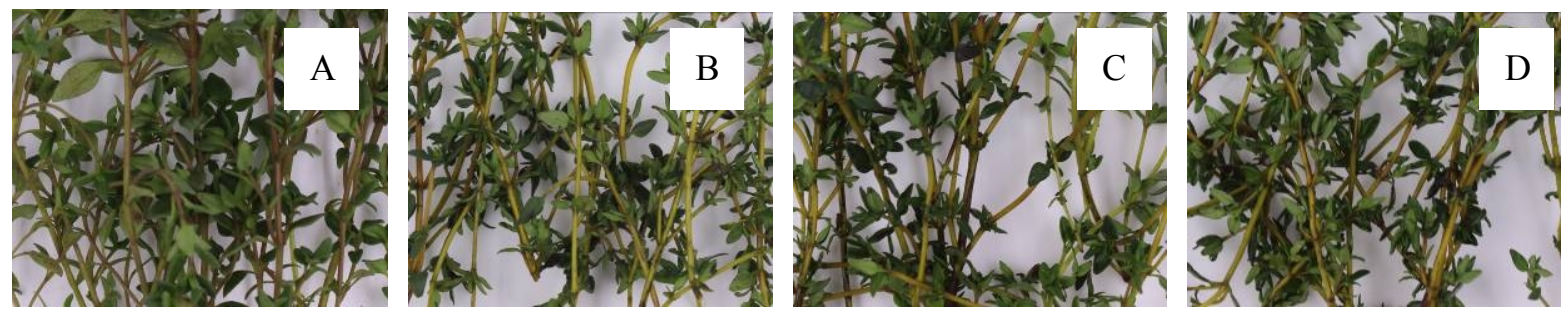

Figure 2 Effect of packaging on the sensory features of the thyme leaf A: fresh thyme; B: Control; C: 1-MCP; D: MAP.

\section{Effect of packaging on chlorophyll content of fresh-cut thyme}

The effect of packaging on the chlorophyll content of the fresh-cut thyme that was stored for $6 \mathrm{~d}$ was evaluated (Figure 3). The chlorophyll content of each treatment was significantly lower than that of the fresh thyme. Mover, the chlorophyll content of the 1-MCP and MAP treatments was higher than that of the control. The chlorophyll content reflected the greenness of the sample (Bellomo \& Fallico, 2007). Hence, the MAP treatment was effective to hold the greenness of the thyme.

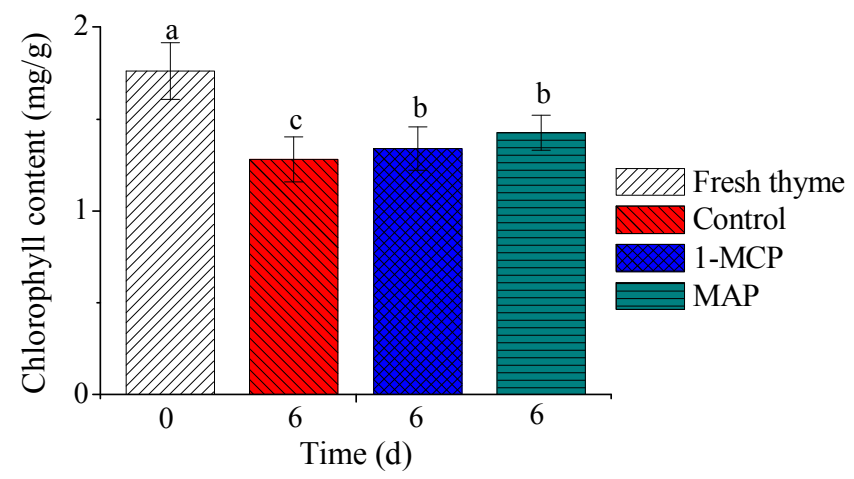

Figure 3 Effect of packaging on the chlorophyll content of fresh-cut thyme

\section{Effect of packaging on flavor of fresh-cut thyme.}

The effect of packaging on the flavor of the fresh-cut thyme that was stored for $6 \mathrm{~d}$ is shown in Figure 4. The principal component analysis showed that the flavor of the sample was mainly 
contributed by the main component 1 and main component 2 . The main component 1 and main component 2 contributed $95.27 \%$ and $4.71 \%$ for the flavor of the fresh-cut thyme. The main component 1 and 2 accounted $99.98 \%$ of the total flavor, which were effective to reflect the flavor of the thyme. Each treatment leaded to a significant flavor variety compared with the fresh thyme. However, the flavor of the MAP and 1-MCP treatments was similar to that of the control. Hence, the MAP and 1-MCP treatments showed no influence on the flavor of the fresh-cut thyme.

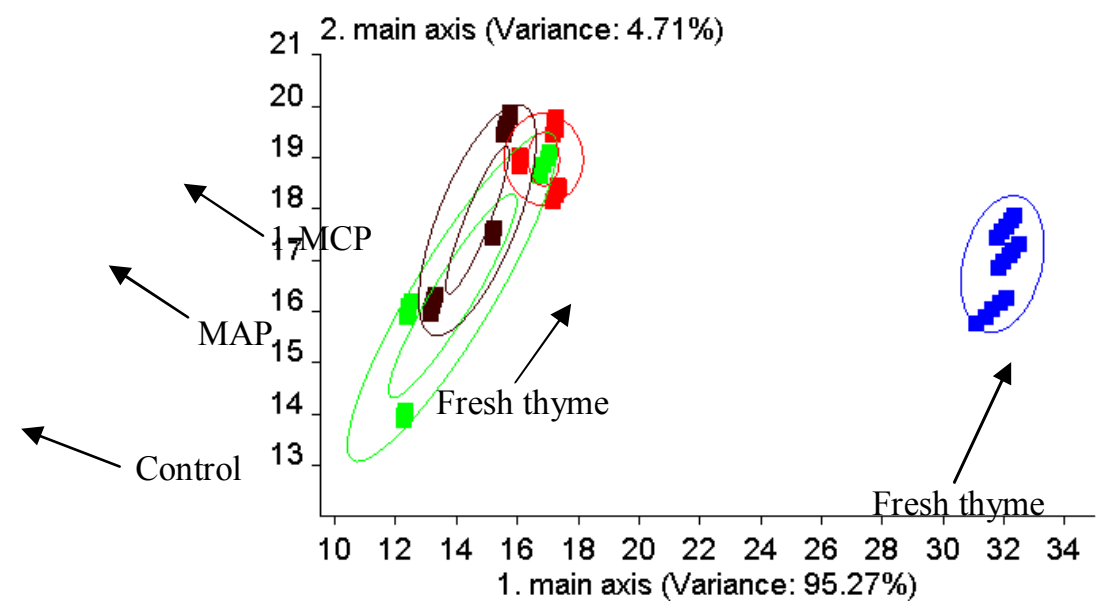

Figure 4 Effect of packaging on the flavor variety of fresh-cut thyme

\section{Conclusions}

The packaging showed no influence on the shelf life of the thyme. The 1-MCP and MAP treatments hold the sensory features of the thyme, and avoid the loss of the greenness of the fresh thyme. Moreover, the 1-MCP and MAP treatment shown no influence of the flavor of the of the fresh-cut thyme.

\section{Acknowledgements}

The authors are grateful to financial support of the earmarked fund for Modern Agro-industry Technology Research System (CARS-26-22 \& CARS-25), Beijing Academy of Agricultural and Forestry Sciences, New Discipline Breeding (KJCX20140204), and Beijing Key Laboratory of Fruits and Vegetable Storage and Processing (Z141105004414037).

\section{References}

[1] Bellomo, M.G. \& Fallico, B. 2007. Anthocyanins, chlorophylls and xanthophylls in pistachio nuts (Pistacia vera) of different geographic origin. Journal of Food Composition and Analysis 20(3-4):352-359.

[2] Bennik, M.H.J., Peppelenbos, H.W., Nguyen-the, C., Carlin, F., Smid, E.J. \& Gorris, L.G.M. 1996. Microbiology of minimally processed, modified-atmosphere packaged chicory endive. Postharvest Biology and Technology 9(2):209-221.

[3] Cefola, M. \& Amodio, L.M. 2010. Exposure to 1-methylcyclopropene (1-MCP) delays the effects of ethylene on fresh-cut broccoli raab (Brassica rapa L.). Postharvest Biology and Technology 58(1):29-35.

[4] Fornari, T., Vicente, G., Vazquez, E., Garcia-Risco, M.R. \& Reglero, G. 2012. Isolation of essential oil from different plants and herbs by supercritical fluid extraction. Journal of Chromatography A 1250(0):34-48.

[5] Gil, M.I., Conesa, M.A. \& Artes, F. 2002. Quality changes in fresh cut tomato as affected by modified atmosphere packaging. Postharvest Biology and Technology 25(2):199-207.

[6] Hershkovitz, V., Saguy, S.I. \& Pesis, E. 2005. Postharvest application of 1-MCP to improve the quality of various avocado cultivars. Postharvest Biology and Technology 37(3):252-264. 
[7] Kulkarni, G.S. \& Vijayanand, P. 2012. Effect of pretreatments on quality characteristics of dehydrated ivy gourd (Coccinia indica L.) Food and Bioprocess Technology 5(2):593-600.

[8] Mathur, P.P., Marshal, A. \& Cheng, A. 2003. Protein profiles in various epidedymal segments of normal and castrated rat. Asian Journal of Andrology 2:57-64.

[9] Omidbeygi, M., Barzegar, M., Hamidi, Z. \& Naghdibadi, H. 2007. Antifungal activity of thyme, summer savory and clove essential oils against Aspergillus flavus in liquid medium and tomato paste. Food Control 18(12):1518-1523.

[10] Rivasa, A., Rodrigoa, D., Martíneza, A., Barbosa-Cánovasb, G.V. \& Rodrigo, M. 2006. Effect of PEF and heat pasteurization on the physical-chemical characteristics of blended orange and carrot juice. LWT 39(10):1163-1170.

[11]Rocculi, P., Cocci, E., Romani, S., Sacchetti, G. \& Rosa, M.D. 2009. Effect of 1-MCP treatment and N2O MAP on physiological and quality changes of fresh-cut pineapple. Postharvest Biology and Technology 51(3):371-377.

[12]Rota, M.C., Herrera, A., Martinez, R.M., Sotomayor, J.A. \& Jordon, M.J. 2008. Antimicrobial activity and chemical composition of Thymus vulgaris, Thymus zygis and Thymus hyemalis essential oils. Food Control 19(7):681-687.

[13] Silveira, A.C., Araneda, C., Hinojosa, A. \& Escalona, V.H. 2014. Effect of non-conventional modified atmosphere packaging on fresh cut watercress (Nasturtium officinale R. Br.) quality. Postharvest Biology and Technology 92(0):114-120.

[14] Vilas-Boas, E.V.d.B. \& Kader, A.A. 2006. Effect of atmospheric modification, 1-MCP and chemicals on quality of fresh-cut banana. Postharvest Biology and Technology 39(2):155-162.

[15] Waghmare, R.B. \& Annapure, U.S. 2013. Combined effect of chemical treatment and/or modified atmosphere packaging (MAP) on quality of fresh-cut papaya. Postharvest Biology and Technology 85:147-153.

[16] Watkins, C.B. 2006. The use of 1-methylcyclopropene (1-MCP) on fruits and vegetables. Biotechnology Advances 24(4):389-409. 